das Spektrum heller Banden zeigt, hauptsächlich gasiger Natur. $\mathrm{Ob}$ sich aber auch einzelne Teile (besonders bei der Umbiegung in der Haube, wo die Moleküle ein Minimum der Geschwindigkeit und ein Maximum der Dichtigkeit erreichen) wieder zu Flüssigkeiten verdichten, die dann schnell gefrieren müßten, ist der Untersuchung wert. In diesem Falle ist das Auftreten kleiner Sternschnuppen im Schweife nicht ausgeschlossen.

Der Schweif als solcher ist natürlich um die Zeit des Durchgangs nicht sichtbar, da er durch perspektivische Verkürzung verloren geht. Holetschek gibt in den Denkschriften der Wiener Akademie, math. Klasse Bd. 63 die Länge des Schweifes des Halleyschen Kometen beim Perihel zu 0.20 und zu 0.15 Sonnenweiten an. Die Erde ist beim Durchgang 0.16 Sonnenweiten vom Kometen entfernt. Der von einem geeigneten Standpunkt außerhalb der Erde sichtbare Schweif würde knapp bis zur Erde reichen, aber seine 'Teilchen würden sie doch treffen.

Nun habe ich die Hyperbelbahnen der Schweifteilchen, zur Kontrolle nach verschiedenen Methoden, berechnet und zwar ephemeridenartig mit Interpolation der Zeiten des Ausgangs und Auftreffens und Prüfung der interpolierten Werte durch direkte Nachrechnung, wobei eine Zeichnung auf Radialpapier die Anschauung erleichterte. Dabei nahm ich die abstoßende Kraft I $-\mu$ zu 4 , I 6 und $6_{4}$ an. Der erstere Wert entspricht den kleinen von Bredichin ermittelten abstoßenden Kräften, I 6 entspricht etwa dem Maximum der abstoßenden Kraft nach Schwarzschild, wenn diese allein vom Lichtdruck herrührt, $6_{4}$ den größeren in letzter Zeit gefundenen Kräften. Pokrowsky fand A. N. 4393 I04, Kopff
A. N. 4348 sogar gegen 2000 . Doch die Berechnung für größere Werte von $\mathrm{I}-\mu$ schien nicht nötig, da für $\mathrm{I}-\mu==\infty$ Ausgang und Auftreffen auf Mai 18.6 fällt. So fand sich nach Cowells und Crommelins Elementen

\begin{tabular}{|c|c|c|c|c|c|c|}
\hline $\begin{array}{l}\text { für } \\
\mathrm{I}-\mu\end{array}$ & $\begin{array}{r}A \\
\text { vom }\end{array}$ & $\begin{array}{l}\text { usgang } \\
\text { Kometen }\end{array}$ & $\begin{array}{c}\mathrm{Au} \\
\text { auf }\end{array}$ & $\begin{array}{l}\text { treffen } \\
\text { lie Erde }\end{array}$ & $\begin{array}{c}\text { also } \\
\text { Wegzeit }\end{array}$ & $\begin{array}{c}\text { Breite } \\
\text { der Erde }\end{array}$ \\
\hline 4 & Mai & 8.306 & Mai & I 9.442 & I I ${ }^{d} 3^{6}$ & $-0^{\circ}$ I $3^{\prime} \cdot 3$ \\
\hline 16 & » & 12.676 & $\gg$ & I9.1 I 5 & 6.439 & $\begin{array}{ll}-0 & 7.9\end{array}$ \\
\hline 64 & " & 15.327 & » & 18.892 & $3.5^{6} 5$ & -0 \\
\hline
\end{tabular}

Die Breite der Erde ist hier von der Kometenbahnebene aus gerechnet.

- Der Radiant etwaiger kleiner Sternschnuppen liegt

\begin{tabular}{|c|c|c|c|}
\hline bei $I-\mu$ & RA. & Dekl. & bei \\
\hline & $\mathrm{o}^{\mathrm{h}} 44^{\mathrm{m}} \cdot 9$ & $+\mathrm{I} 8^{\circ} 7^{\prime}$ & scium \\
\hline I 6 & I 28.0 & +1929 & scium \\
\hline 64 & 210.4 & $+20 \mathrm{II}$ & Arietis \\
\hline
\end{tabular}

Da bei dem Bielaschen Kometen 1872 und 1885 der Radiant mit der Bahn übereinstimmte, so ging die Erde durch den kugeligen Haufen, und nicht durch den Schweif, wie oft angenommen wird. Wäre sie durch den Schweif gegangen, so wäre der Radiant anders gewesen.

Kleine Planeten, obgleich wie die Kometen ohne Atmosphäre, bilden deshalb keinen Schweif, weil die rerdampfbaren Stoffe auf ihrer Oberfäche längst aufgebraucht sind. Würde ein solcher aber, etwa durch Zusammenstoß, in Teile zerfallen, so würden verdampfbare Stoffe aus dem Inneren Ausströmungen und Schweif bilden, der freilich bei der geringen Sonnennähe klein bleibt.

Breslau, I9 10 April I 2.

F. Franz.

\title{
Beobachtungen des Halleyschen Kometen 1909 c.
}

I 9 Io April 20 I $6^{\mathrm{h}} 31^{\mathrm{m}}$ I $2^{\mathrm{s}} \mathrm{t}$. m. Arcetri $d \alpha=+30.43$ $\mathscr{d}=+3^{\prime} \mathrm{I}_{7 \prime}^{\prime \prime}{ }_{4}$ Cfr. I 2,8 « app. $=23^{\mathrm{h}} 5 \mathrm{I}^{\mathrm{m}} 30.8 \mathrm{I}(9.627 \mathrm{n})$ $\delta$ app. $=+7^{\circ} 46^{\prime} 29^{\prime \prime} 6(0.769)$ Red.ad l.app. -1 s.r $5-8.9$.

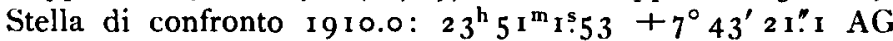
L.pz II 118 I8.

A. Abetti.

I 9 1 0 April $2215^{\mathrm{h}} 54^{\mathrm{m}} 38^{\mathrm{s}}$ M.Z. Berged. $\Delta \alpha=-$ I $7^{\mathrm{s}} .86$ ${ }_{\alpha} / \delta=+3^{\prime} 6^{\prime \prime} .4$ Vgl. $3 \alpha$ app. $=23^{\mathrm{h}} 50^{\mathrm{m}} 42^{\mathrm{s}} \cdot 56(9.548 \mathrm{n})$

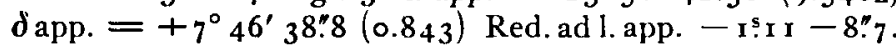

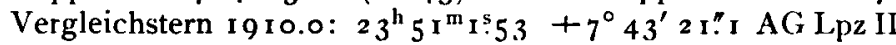
I 8 I 8 . Kometenkern etwa $4^{\mathrm{m}}$, mit einem hellen Ausläufer o'5 lang im PW I $25^{\circ}$. Ije Begrenzung des $4^{\prime}$ breiten Schweifes konnte bis $3^{\prime}$ vom Kern verfolgt werden, PW des Schweifes $270^{\circ}$.

H. Thiele.

Der Halleysche Komet wurde auf der Sternwarte Tasch- kent an den Abenden I9 10 Febr. 28, März I und 2 mit einem Zeiß-Planar (Lichtstärke $1: 3.6$ ) photographiert. Der Komet zeigt einen Schweif von etwa $0^{\circ} .5$ Länge. Die Richtung des Schweifes nach OSO bildet mit der Richtung der Bewegung des Kometen einen Winkel von etwa $25^{\circ}$.

F. Sykora.

Der Halleysche Komet erschien 19 10 April I 7 im dreizölligen Kometensucher der Sternwarte Breslau wie ein matter hellgelber Fleck in der dunkelgelben Morgendämmerung. April 23 sah ich ihn wieder von $15^{\mathrm{h}} 4^{\mathrm{I}^{\mathrm{m}} \mathrm{m}}$. Z. Breslau an. Er war viel heller, $2^{m} \cdot 9$, und stimmt mit Ebells Vorausberechnung $H$, nicht $(H)$, in A. N. 4400. Deutlicher Nebel ohne Schweif, weißlichgelb auf grünlichem Hintergrunde, etwas länglich der Sonne zu, mit Haube.

F. Franz.

\section{Anomalien auf Jupiter.}

Von Ph. Fauth.

Die Überwachung der Umgebung des ehemals roten Großen Flecks auf Jupiter dürfte jetzt besonders notwendig sein, weil sich da merkwürdig stark ausgeprägte Vorgänge im Strömen der Materie verraten. Niemals möchte deutlicher geworden sein, daß der ovale Fleck der direkten Eigenbewegung des dunklen Stoffes in $\operatorname{der} \operatorname{STr} Z$ ein Hindernis bietet, als Ende März ig ro.

Am 27. März lag die Spitze der Rechten Schulter in 